\title{
Eicosanoid Production
}

National Cancer Institute

\section{Source}

National Cancer Institute. Eicosanoid Production. NCI Thesaurus. Code C40652.

Eicosanoid Production consists of synthesis of hormone-like endogenous unsaturated and oxygenated fatty acid derivatives of arachidonic acid (eicosanoids) that act near the site of synthesis and have a specific regulatory effect on the activity of target cells in host defense reactions, immediate hypersensitivity, and inflammation. Eicosanoids include prostaglandins, leukotrienes, thromboxanes, and hydroxyeicosatetraenoic acid compounds. 